\title{
Plasma Orexin-A Levels in COPD Patients with Hypercapnic Respiratory Failure
}

\author{
Lin-Yun Zhu, ${ }^{1}$ Hanssa Summah, ${ }^{2}$ Hong-Ni Jiang, ${ }^{1}$ and Jie-Ming $\mathbf{Q u}{ }^{2}$ \\ ${ }^{1}$ Department of Pulmonary Medicine, Zhongshan Hospital, Fudan University School of Medicine, Shanghai 200032, China \\ ${ }^{2}$ Department of Pulmonary Medicine, Huadong Hospital, Fudan University School of Medicine, Shanghai 200040, China
}

Correspondence should be addressed to Hong-Ni Jiang, jhn2001@sina.com and Jie-Ming Qu, jmqu64@yahoo.com.cn

Received 15 January 2011; Revised 12 March 2011; Accepted 1 April 2011

Academic Editor: E. Moilanen

Copyright (C) 2011 Lin-Yun Zhu et al. This is an open access article distributed under the Creative Commons Attribution License, which permits unrestricted use, distribution, and reproduction in any medium, provided the original work is properly cited.

\begin{abstract}
Orexins have previously been shown to promote wakefulness, regulate lipid metabolism and participate in energy homeostasis. The aim of the study was to determine the relationship between plasma orexin-A and body composition in COPD in-patients with hypercapnic respiratory failure. 40 patients with hypercapnic respiratory failure and 22 healthy individuals were enrolled prospectively in this study. Plasma orexin-A levels, $\mathrm{BMI}, \mathrm{SaO}_{2}, \mathrm{PaCO}_{2}$ and $\mathrm{PaO}_{2}$ were noted for all the patients. Plasma orexin-A levels were higher in the underweight (UW) group, normal weight (NW) group and overweight (OW) group of COPD patients as compared with UW, NW and OW group of the control group $(P<.05)$. Plasma orexin-A in COPD patients were higher in the OW group than in the NW group and the UW group. Plasma orexin-A levels showed significant correlation with body mass index (BMI), independent of $\mathrm{PaO}_{2}(r=0.576 ; P<.05)$ and \%fat $(r=0.367 ; P<.05)$; a negative correlation was noted between plasma orexin-A levels and $\mathrm{PaO}_{2}(r=-0.738 ; P<.05)$ and $\mathrm{SaO}_{2}(r=-0.616 ; P<.05)$. Our results suggest that orexin-A levels are high in COPD patients with hypercapnic respiratory failure, and vary according to BMI and body composition. Orexin-A may be associated with the severity of hypoxemia in COPD patients with hypercapnic respiratory failure.
\end{abstract}

\section{Introduction}

Body weight is an important prognostic factor of chronic obstructive pulmonary disease in that a low body weight correlates with increased morbidity and poor prognosis [1-3]. This is mainly because a low body weight in COPD patients implies weakening of both respiratory muscles and skeletal muscles $[4,5]$. The weakening of respiratory muscles, in turn, makes COPD patients vulnerable to respiratory failure. It is widely known that COPD is one of the main causes of acute respiratory failure. Resting hypoxia, usually due to $V_{A} / Q$ mismatching, and exercise aggravation of hypoxia due to increased flow and oxygen disequilibrium are often seen in COPD patients $[6,7]$. Acute hypercapnic respiratory failure as a result of an acute exacerbation of COPD is a common reason for emergency hospital admission [8]. It has been noted that an acute exacerbation of COPD entails decreased food intake and increased resting energy expenditure, thus causing energy disbalance in these patients [9].

Orexin-A and orexin-B belong to the family of orexins are neuropeptides identified within the lateral hypothalamus during the last decade [10]. Previous studies show that intraventricular injection of orexin-A not only produces a dose-dependent food intake but also increases the metabolic rate $[10,11]$. Apart from promoting wakefulness and regulating lipid metabolism, orexin-A has been implicated in diabetes mellitus and obesity [12-14]. In 2003, Matsumura et al. successfully showed that plasma orexin-A levels are lower in COPD patients, with a lower level being more prominent in underweight patients as compared to normal individuals [15]. However, whether plasma orexin-A levels show the same variation in COPD patients with hypercapnic respiratory failure has not been investigated. We therefore tried to assess plasma orexin-A levels during an acute exacerbation of COPD.

\section{Materials and Methods}

2.1. Subjects. Forty COPD patients with hypercapnic respiratory failure who were hospitalized in the Department of Pulmonary Medicine of Zhongshan Hospital, Shanghai, were included in the study. The diagnosis of COPD was 
based on past smoking history, clinical evaluation, and pulmonary function tests according to the American Thoracic Society Guidelines [16]. All the 40 patients had an arterial partial pressure of $\mathrm{CO}_{2}\left(\mathrm{PaCO}_{2}\right)$ of $\geq 50 \mathrm{mmHg}$, which is line with hypercapnic respiratory failure diagnosis. Patients with a history of use of systemic corticosteroids or with a history of sleep-related breathing disorders, malignancy, cardiac failure, diabetes mellitus, or other endocrine diseases were excluded. The control group consisted of 22 healthy individuals, without any underlying disease and with normal pulmonary function as defined by $\left(\mathrm{FEV}_{1} / \mathrm{FVC}>70 \%\right)$ who presented to the Zhongshan Hospital for routine physical examination. The research protocol was approved by the Ethics Committee of Fudan University, and written consent form was obtained for each subject.

2.2. Nutritional Assessment. The following parameters were recorded for each patient: age (in years), body weight (in kilograms), and height (in meters). Based on these measures, body mass index (BMI) and body fat percentage were calculated. BMI was calculated as weight $/(\text { height })^{2}$. Body fat $\%$ was calculated as follows: male $=(1.2 \times \mathrm{BMI})+(0.23 \times$ age $)-16.2$, and female $=(1.2 \times \mathrm{BMI})+(0.23 \times$ age $)-$ 5.4 .

Based in their BMI, the control group subjects and the patients with COPD were further subdivided into 3 groups: (1) underweight (UW) group (BMI < 20), (2) normal weight (NW) group $(20 \leq \mathrm{BMI}<25)$, and (3) overweight (OW) group (BMI $\geq 25)$.

2.3. Samples. $4 \mathrm{~mL}$ blood samples were collected from the antecubital vein by using a vacuum blood-collecting tube containing aprotinin at noon. Plasma, separated by centrifugation, was stored at $-80^{\circ} \mathrm{C}$ until analysis. Orexin-A concentrations were always measured in duplicate in the following way: plasma orexin-A was first extracted using extraction columns (Extra-Sep C18, Lida, Kenosha, Wisc., USA). Then, column eluants were evaporated to dryness in a centrifugal concentrator and reconstituted in radioimmunoassay buffer. Finally, orexin-A levels in the reconstituted aliquots were determined using an iodine-125 hypocretin-1 RIA system (Phoenix Pharmaceuticals, Mountain View, Calif, USA). Mean values of orexin-A were calculated and used for statistical analysis.

Arterial blood was drawn from all subjects during room air breathing at the same time that blood was collected for orexin-A level measurement.

2.4. Statistical Analysis. The Mann-Whitney $U$-test and ANOVA were used to analyze the difference between two groups. Spearman's rank correlation technique and partial correlation technique were used to analyze the relationship between several continuous variables. Results are expressed as medians [range] or mean \pm SE as appropriate. Statistical analysis was performed using a statistical software package (SPSS 13.0); $P<.05$ was considered to be statistically significant.
TABLE 1: Baseline characteristics of subjects.

\begin{tabular}{lcccc}
\hline \multicolumn{1}{c}{ Group } & & $n$ & Age (years) & BMI $\left(\mathrm{kg} / \mathrm{m}^{2}\right)$ \\
\hline \multirow{4}{*}{ Control subjects } & UW & 5 & $73.8 \pm 11.9$ & $19.2 \pm 0.9$ \\
& NW & 14 & $72.6 \pm 10.4$ & $22.0 \pm 1.1$ \\
& OW & 3 & $74.0 \pm 8.7$ & $28.5 \pm 2.4$ \\
\hline \multirow{3}{*}{ COPD } & UW & 10 & $76.2 \pm 5.0$ & $18.3 \pm 1.2$ \\
& NW & 23 & $73.2 \pm 10.4$ & $21.7 \pm 1.4$ \\
& OW & 7 & $74.7 \pm 7.0$ & $26.2 \pm 0.9$ \\
\hline
\end{tabular}

TABle 2: Plasma orexin-A levels in weight-based group.

\begin{tabular}{cccccccc}
\hline & \multicolumn{3}{c}{ Control subjects } & \multicolumn{4}{c}{ Patients with COPD } \\
& \multicolumn{2}{c}{$\begin{array}{c}\text { orexin-A }(\mathrm{pg} / \mathrm{mL}) \\
\text { median }\end{array}$} & range & $n$ & \multicolumn{2}{c}{ orexin-A (pg/mL) } & $P$ \\
& & median & range & \\
\hline UW & 5 & 9.00 & $8.00-18.69$ & 10 & 36.32 & $22.71-44.88$ & .000 \\
NW & 14 & 15.44 & $5.96-33.61$ & 23 & 48.51 & $14.55-95.86$ & .000 \\
OW & 3 & 20.55 & $10.18-23.62$ & 7 & 56.00 & $45.26-101.26$ & .001 \\
\hline
\end{tabular}

\section{Results}

3.1. Subject Characteristics. Out of the 40 patients with COPD, 32 were male and 8 were female. The mean age of the subjects was $(74.2 \pm 8.7)$ years and the mean BMI was (21.7 \pm $3.0) \mathrm{kg} / \mathrm{m}^{2}$. Out of the 22 control subjects, 17 were male and 5 were female. The mean age was $(73.1 \pm 10.1)$ years and the mean BMI was $(22.3 \pm 3.0) \mathrm{kg} / \mathrm{m}^{2}$. The mean age and the mean BMI of COPD patients and control subjects belonging to the UW, NW, and OW groups are shown in Table 1. There was no statistical difference in the baseline characteristics (age and BMI) between the patients with COPD and the control subjects.

3.2. Plasma Orexin-A Levels. Plasma orexin-A levels in each group of COPD patients and control subjects are shown in Table 2. UW, NW, and OW patients with COPD had significantly higher plasma orexin-A levels as compared to UW, NW, and OW control subjects $(P<.000, P<.000$, and $P<.001$, resp.).

\subsection{Relationship between Different Observed Indexes of COPD} Patients and Control Subjects and Plasma Orexin-A Levels. Plasma orexin-A levels in COPD patients were found to correlate significantly with BMI $(r=0.589, P=.000)$ (Figure 1(a)), \%fat $(r=0.367, P=.020)$ (Figure 1(b)), $\mathrm{PaO}_{2}(r=-0.738, P=.000)$ (Figure $\left.1(\mathrm{c})\right)$, and $\mathrm{SaO}_{2}(r=$ $-0.616, P=.000$ ) (Figure 1(d)). Partial correlation analysis was used to determine whether the correlation between plasma orexin-A and BMI was independent of the degree of respiratory failure, and, controlling $\mathrm{PaO}_{2}$, plasma orexin- $\mathrm{A}$ was still found to correlate significantly with BMI $(r=0.576$, $P=.000)$. However, no correlation was found between $\mathrm{PaCO}_{2}$ and plasma orexin-A levels $(P=.173)$.

Plasma orexin-A levels in control subjects were found to correlate significantly with BMI $(r=0.428, P=.047)$. 


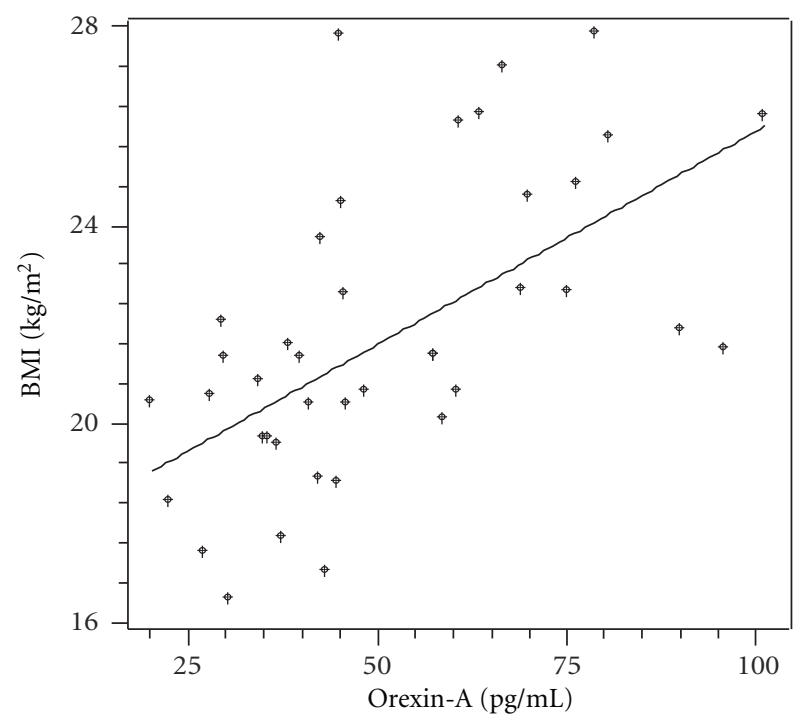

(a)

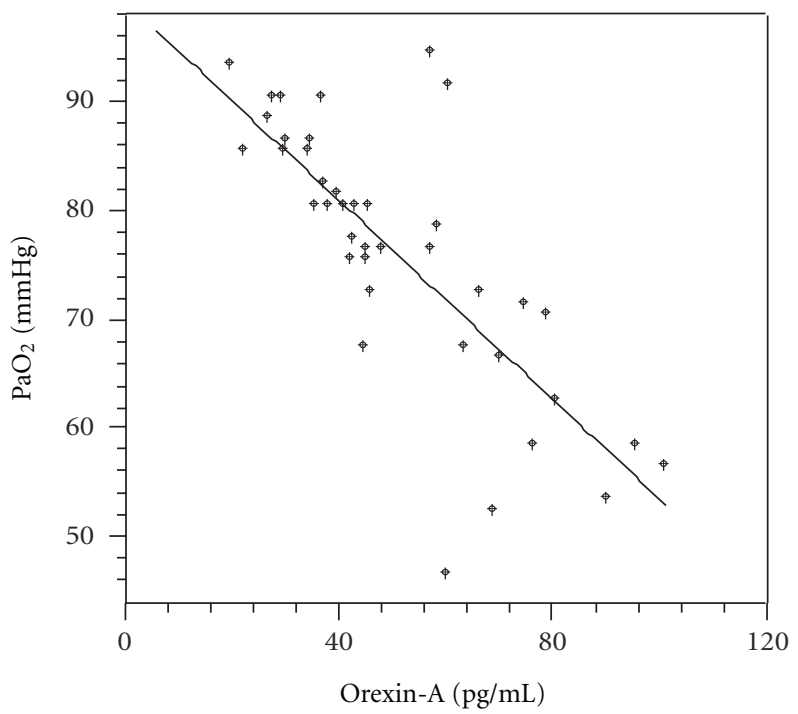

(c)

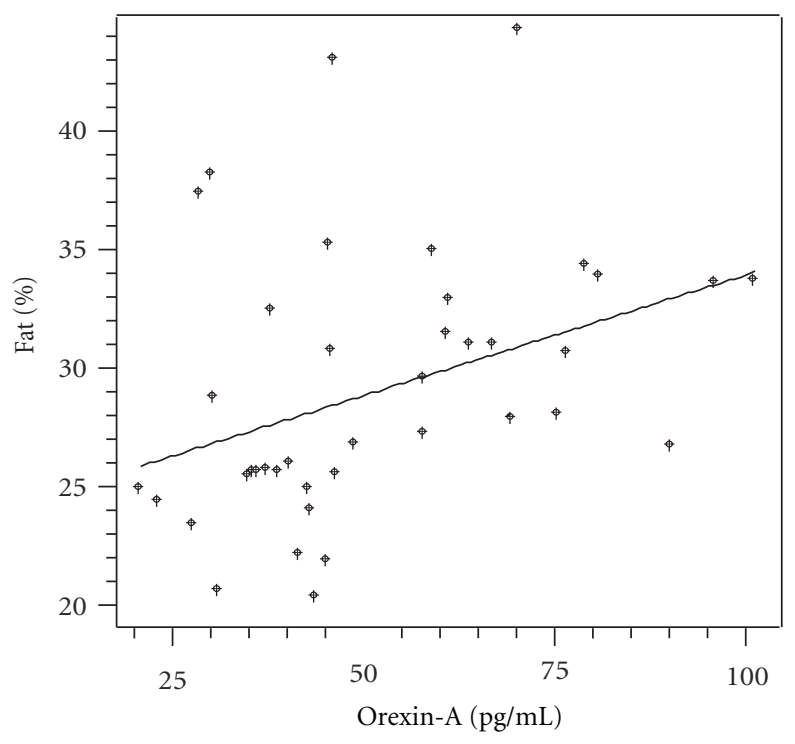

(b)

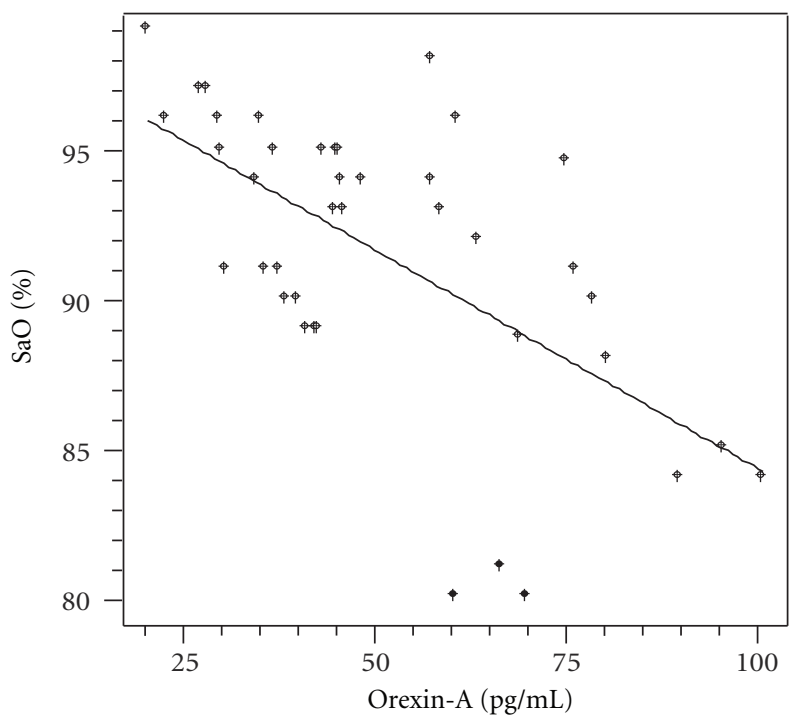

(d)

Figure 1: (a) Relationship between BMI and orexin-A. (b) Relationship between \%fat and orexin-A. (c) Relationship between PaO $\mathrm{O}_{2}$ and orexin-A. (d) Relationship between $\mathrm{SaO}_{2}$ and orexin-A. In patients with COPD, plasma orexin-A levels significantly correlated with BMI $(r=0.589, P=.000), \%$ fat $(r=0.367, P=.020), \mathrm{PaO}_{2}(r=-0.738, P=.000), \mathrm{SaO}_{2}(r=-0.616, P=.000)$.

\section{Discussion}

Orexin, also known as hypocretin, was first described in 1998 by de Lecea et al. [17]. In 1999, orexin neurons were found to be present in the lateral hypothalamic area, perifornical nucleus, diffuse part of the dorsomedial hypothalamic nucleus, and posterior hypothalamus [18]. It has been previously shown that orexins can pass the bloodbrain barrier by simple diffusion [19] and that orexins and orexins receptors are present in the hypothalamus as well as the enteric nervous system, adipose tissue, the pancreas and the gut $[20,21]$. Orexin not only acts as an appetite stimulator but also acts along with other neuropeptides to regulate feeding behavior $[22,23]$.
Our results show that plasma orexin-A levels in COPD patients with hypercapnic respiratory failure are much higher than those in normal subjects. These results differ from those of Matsumura et al. [15]. We can explain the discrepancies in our results by the fact that our study included a larger number of patients and our patients were all hospitalized COPD patients with hypercapnic respiratory failure while those of Matsumura et al. were outpatients, without any exacerbation of COPD. COPD patients with hypercapnic respiratory failure have a larger amount of energy expenditure and have decreased food intake as compared to COPD patients who are not suffering from an acute disease exacerbation. This energy imbalance may well be another plausible explanation for the higher values of 
plasma orexin-A in COPD patients in our study. Due to the fact that COPD patients with hypercapnic respiratory failure restricted food intake, there is subsequently an increase in the expression of prepro-orexin gene [24], which in turn causes an increase in the plasma orexin-A levels noted in our patients.

Patients from the OW group were found to have higher plasma orexin-A levels than those from the NW group, who in turn, had higher plasma orexin-A levels than those of the UW group. We further found that plasma orexin-A levels and BMI were correlated and the correlation was independent of the degree of respiratory failure. These results are in concordance with those of Matsumura et al. Other previous studies have shown that plasma orexin-A levels correlate with BMI $[25,26]$. However, Adam et al. found that plasma orexin-A was lower in obese individuals [27]. These different results might well be explained by the fact that plasma orexin-A levels might be dependent on the underlying disease from which the patients are suffering from. In our present study, we also found a positive correlation between plasma orexin-A levels and \%fat in COPD patients with hypercapnic respiratory failure. In 2002, Wortley et al. showed that circulating lipids increase in obesity and the increase in triglyceride levels increase hypothalamic orexin gene expression in rats [28]. In 2006, Digby et al. showed that orexin receptors are found in adipose tissue and that orexins may play a role in adipogenesis [21]. Therefore, our results are in concordance with these findings.

Orexin participates in respiratory control by increasing ventilation [29]. Dreshaj et al. showed that lesions in the basomedial hypothalamus have been found to influence respiratory response in neonatal rats in that the latter show impaired response to hypoxia. However, these lesions did not affect the hypercapnic responses [30]. On the other hand, Deng et al. showed that orexin knock-out mice had weakened respiratory chemoreflex to hypercapnic gases, but not to hypoxic gases [31]. In our study, we found no correlation between plasma orexin-A levels and $\mathrm{PaCO}_{2}$; but, a significant negative correlation was noted between plasma orexin-A levels and $\mathrm{PaO}_{2}$ and $\mathrm{SaO}_{2}$. Similar findings have been formerly noted [32]. Our results indicate that orexinA may play an important part during hypoxia; however, the underlying mechanism remains to be revealed.

Here we must point out that there are several limitations to our study. Firstly, we included a limited number of patients. Although plasma orexin-A levels in COPD patients were found to correlate significantly with $\mathrm{BMI}$ \% fat, $\mathrm{PaO}_{2}$ and $\mathrm{SaO}_{2}$, the results for individual COPD patients were in fact, quite variable, and therefore, studies including larger number of patients should be carried out in order to confirm our results. Moreover, in our study we did not study the mechanism through which orexin-A is upregulated during hypoxia.

\section{Conclusions}

This study shows that plasma orexin-A levels correlate with $\mathrm{BMI}$ and \%fat in COPD patients with hypercapnic respiratory failure and that orexin-A levels may be altered during hypoxia, and more studies are required to determine the role of orexin-A in hypoxia.

\section{Conflict of Interest}

None of the authors have any conflict to disclose.

\section{Acknowledgment}

This study was supported by the National Basic Research Program of China (973 Program) "Study of Gut Microbiome and Infections" 2007CB513000 and the "Shu Guang" Sequel Program of Shanghai Education Commission, China (no. 01SG06) and Shanghai Leading Talent Projects (no. 036, 2010). Lin-Yun Zhu and Hanssa Summah are contributed equally to this work.

\section{References}

[1] C. Landbo, E. Prescott, P. Lange, J. Vestbo, and T. P. Almdal, "Prognostic value of nutritional status in chronic obstructive pulmonary disease," American Journal of Respiratory and Critical Care Medicine, vol. 160, no. 6, pp. 1856-1861, 1999.

[2] D. O. Wilson, R. M. Rogers, E. C. Wright, and N. R. Anthonisen, "Body weight in chronic obstructive pulmonary disease. The National Institutes of Health Intermittent Positive-Pressure Breathing Trial," American Review of Respiratory Disease, vol. 139, no. 6, pp. 1435-1438, 1989.

[3] A. M. W. J. Schols, J. Slangen, L. Volovics, and E. F. M. Wouters, "Weight loss is a reversible factor in the prognosis of chronic obstructive pulmonary disease," American Journal of Respiratory and Critical Care Medicine, vol. 157, no. 6, part 1, pp. 1791-1797, 1998.

[4] M. A. P. Vermeeren, E. C. Creutzberg, A. M. Schols et al., "Prevalence of nutritional depletion in a large out-patient population of patients with COPD," Respiratory Medicine, vol. 100, no. 8, pp. 1349-1355, 2006.

[5] M. P. K. Engelen, A. M. Schols, W. C. Baken, G. J. Wesseling, and E. F. M. Wouters, "Nutritional depletion in relation to respiratory and peripheral skeletal muscle function in outpatients with COPD," European Respiratory Journal, vol. 7, no. 10, pp. 1793-1797, 1994.

[6] R. Rodríguez-Roisin and J. Roca, "Mechanisms of hypoxemia," in Applied Physiology in Intensive Care Medicine, pp. 25-27, Springer, Berlin, Germany, 2006.

[7] W. C. Miller, J. G. Heard, and K. M. Unger, "Enlarged pulmonary arteriovenous vessels in COPD. Another possible mechanism of hypoxemia," Chest, vol. 86, no. 5, pp. 704-706, 1984.

[8] N. Shahrizaila, W. S. Lim, D. K. Robson, W. J. Kinnear, and A. J. Wills, "Tubular aggregate myopathy presenting with acute type II respiratory failure and severe orthopnoea," Thorax, vol. 61, no. 1, pp. 89-90, 2006.

[9] M. A. P. Vermeeren, A. M. Schols, and E. F. M. Wouters, "Effects of an acute exacerbation on nutritional and metabolic profile of patients with COPD," European Respiratory Journal, vol. 10, no. 10, pp. 2264-2269, 1997.

[10] T. Sakurai, A. Amemiya, M. Ishii et al., "Orexins and orexin receptors: a family of hypothalamic neuropeptides and Gprotein-coupled receptor that regulate feeding behavior," Cell, vol. 92, no. 4, pp. 573-585, 1998. 
[11] M. Lubkin and A. Stricker-Krongrad, "Independent feeding and metabolic actions of orexins in mice," Biochemical and Biophysical Research Communications, vol. 253, no. 2, pp. 241245, 1998.

[12] J. G. Sutcliffe and L. de Lecea, "The hypocretins: setting the arousal threshold," Nature Reviews Neuroscience, vol. 3, no. 5, pp. 339-349, 2002.

[13] L. A. Campfield and F. J. Smith, "The pathogenesis of obesity," Best Practice \& Research Clinical Endocrinology \& Metabolism, vol. 13, no. 1, pp. 13-30, 1999.

[14] B. D. Wilson, D. Bagnol, C. B. Kaelin et al., "Physiological and anatomical circuitry between agouti-related protein and leptin signaling," Endocrinology, vol. 140, no. 5, pp. 2387-2397, 1999.

[15] T. Matsumura, M. Nakayama, H. Satoh, A. Naito, K. Kamahara, and K. Sekizawa, "Plasma orexin-A levels and body composition in COPD," Chest, vol. 123, no. 4, pp. 1060-1065, 2003.

[16] American Thoracic Society, "Standards for the diagnosis and care of patients with chronic obstructive pulmonary disease," American Journal of Respiratory and Critical Care Medicine, vol. 152, no. 5, part 2, pp. S77-S121, 1995.

[17] L. de Lecea, T. S. Kilduff, C. Peyron et al., "The hypocretins: hypothalamus-specific peptides with neuroexcitatory activity," Proceedings of the National Academy of Sciences of the United States of America, vol. 95, no. 1, pp. 322-327, 1998.

[18] T. Nambu, T. Sakurai, K. Mizukami, Y. Hosoya, M. Yanagisawa, and K. Goto, "Distribution of orexin neurons in the adult rat brain," Brain Research, vol. 827, no. 1-2, pp. 243-260, 1999.

[19] A. J. Kastin and V. Akerstrom, "Orexin A but not orexin B rapidly enters brain from blood by simple diffusion," Journal of Pharmacology and Experimental Therapeutics, vol. 289, no. 1, pp. 219-223, 1999.

[20] A. L. Kirchgessner and M. T. Liu, "Orexin synthesis and response in the gut," Neuron, vol. 24, no. 4, pp. 941-951, 1999.

[21] J. E. Digby, J. Chen, J. Y. Tang, H. Lehnert, R. N. Matthews, and H. S. Randeva, "Orexin receptor expression in human adipose tissue: effects of orexin-A and orexin-B," Journal of Endocrinology, vol. 191, no. 1, pp. 129-136, 2006.

[22] H. Ganjavi and C. M. Shapiro, "Hypocretin/Orexin: a molecular link between sleep, energy regulation, and pleasure," Journal of Neuropsychiatry and Clinical Neurosciences, vol. 19, no. 4, pp. 413-419, 2007.

[23] T. Moriguchi, T. Sakurai, T. Nambu, M. Yanagisawa, and K. Goto, "Neurons containing orexin in the lateral hypothalamic area of the adult rat brain are activated by insulin-induced acute hypoglycemia," Neuroscience Letters, vol. 264, no. 1-3, pp. 101-104, 1999.

[24] Y. Yamamoto, Y. Ueta, R. Serino, M. Nomura, I. Shibuya, and H. Yamashita, "Effects of food restriction on the hypothalamic prepro-orexin gene expression in genetically obese mice," Brain Research Bulletin, vol. 51, no. 6, pp. 515-521, 2000.

[25] M. V. Heinonen, A. K. Purhonen, P. Miettinen et al., "Apelin, orexin-A and leptin plasma levels in morbid obesity and effect of gastric banding," Regulatory Peptides, vol. 130, no. 1-2, pp. 7-13, 2005.

[26] Y. Kawada, H. Hayashibe, K. Asayama et al., "Plasma levels of orexin-A and leptin in obese children," Clinical Pediatric Endocrinology, vol. 13, no. 1, pp. 47-53, 2004.

[27] J. A. Adam, P. P. C. A. Menheere, F. M. H. Van Dielen, P. B. Soeters, W. A. Buurman, and J. W. M. Greve, "Decreased plasma orexin-A levels in obese individuals," International Journal of Obesity, vol. 26, no. 2, pp. 274-276, 2002.
[28] K. E. Wortley, G. Q. Chang, Z. Davydova, and S. F. Leibowitz, "Peptides that regulate food intake: orexin gene expression is increased during states of hypertriglyceridemia," American Journal of Physiology, vol. 284, no. 6, pp. R1454-R1465, 2003.

[29] J. K. Young, M. Wu, K. F. Manaye et al., "Orexin stimulates breathing via medullary and spinal pathways," Journal of Applied Physiology, vol. 98, no. 4, pp. 1387-1395, 2005.

[30] I. A. Dreshaj, M. A. Haxhiu, R. J. Martin, and J. K. Young, "The basomedial hypothalamus modulates the ventilatory response to hypoxia in neonatal rats," Pediatric Research, vol. 53, no. 6, pp. 945-949, 2003.

[31] B. S. Deng, A. Nakamura, W. Zhang, M. Yanagisawa, Y. Fukuda, and T. Kuwaki, "Contribution of orexin in hypercapnic chemoreflex: evidence from genetic and pharmacological disruption and supplementation studies in mice," Journal of Applied Physiology, vol. 103, no. 5, pp. 1772-1779, 2007.

[32] N. Igarashi, K. Tatsumi, A. Nakamura et al., "Plasma orexin-A levels in obstructive sleep apnea-hypopnea syndrome," Chest, vol. 124, no. 4, pp. 1381-1385, 2003. 


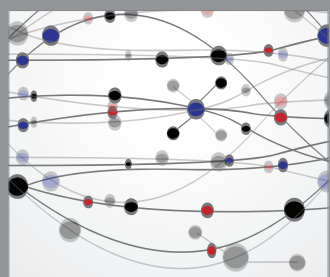

The Scientific World Journal
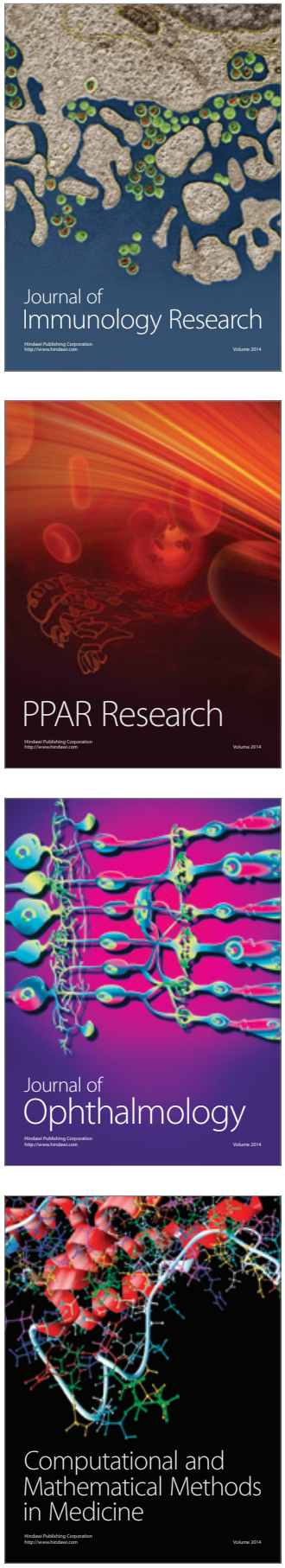

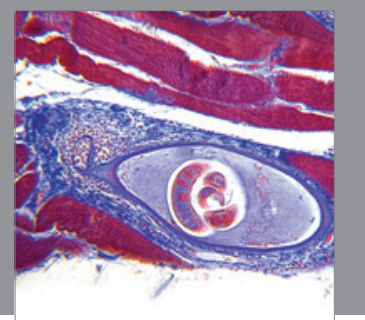

Gastroenterology

Research and Practice
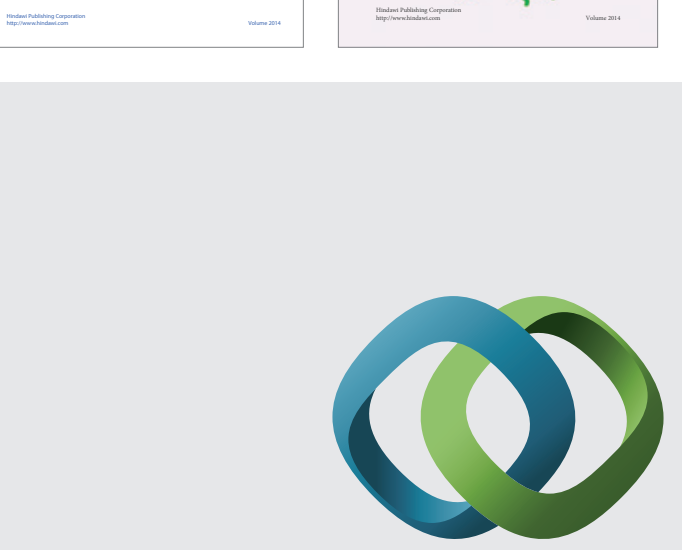

\section{Hindawi}

Submit your manuscripts at

http://www.hindawi.com
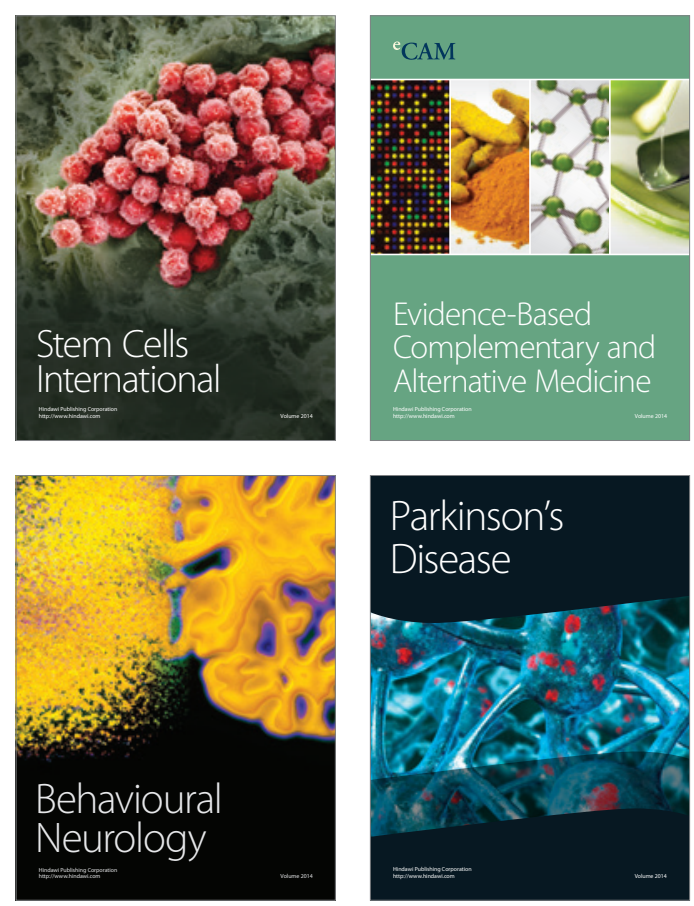

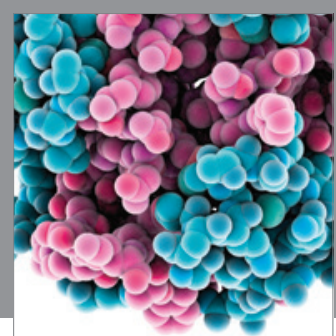

Journal of
Diabetes Research

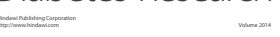

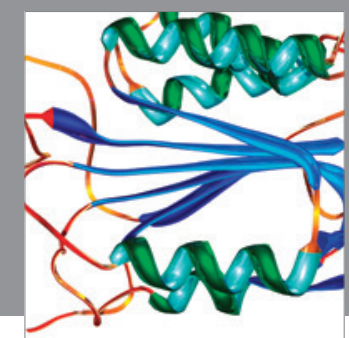

Disease Markers
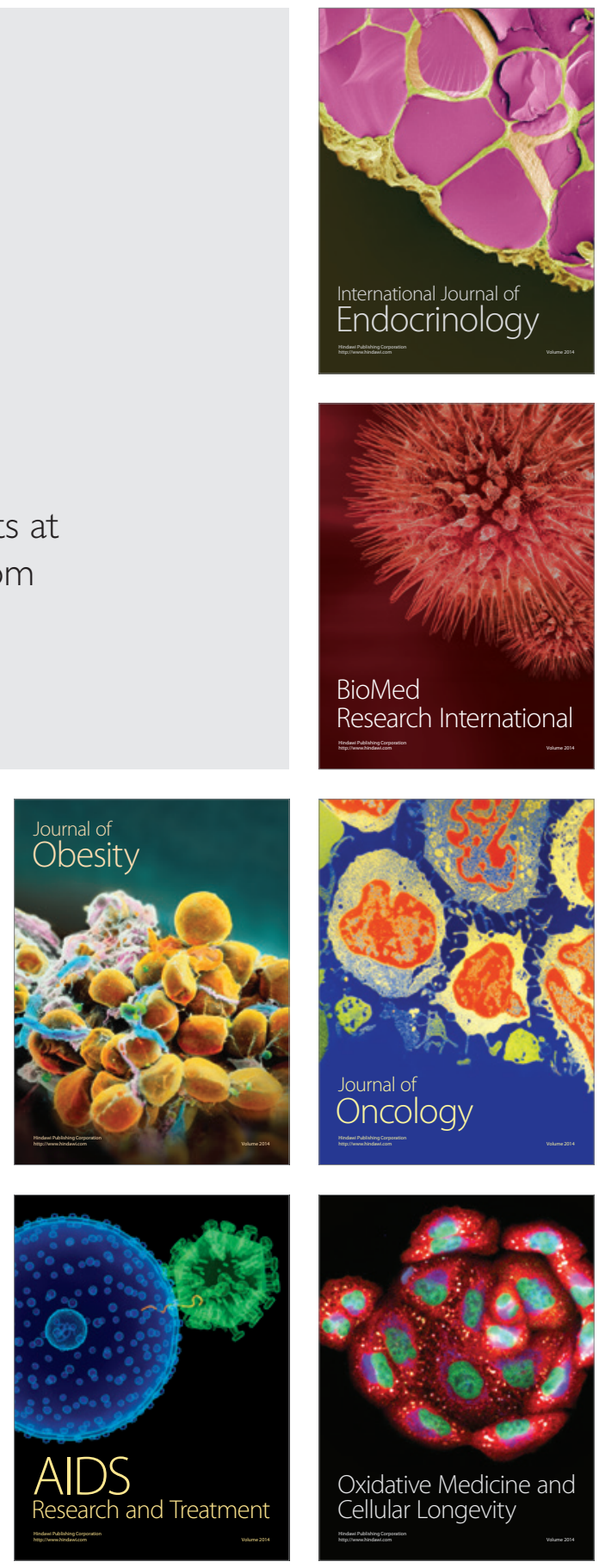